\title{
Properties of bcr-abl-transformed mouse 12B1 cells secreting interleukin-2 and granulocyte-macrophage colony stimulating factor (GM-CSF): II. Adverse effects of GM-CSF
}

\author{
MARTINA PETRÁČKOVÁ ${ }^{1}$, LIBOR STANĚK ${ }^{1,2}$, VÁCLAV MANDYS ${ }^{3}$, PAVEL DUNDR $^{2}$ and VLADIMÍR VONKA ${ }^{1}$ \\ ${ }^{1}$ Department of Experimental Virology, Institute of Hematology and Blood Transfusion; ${ }^{2}$ Institute of Pathology, General \\ Faculty Hospital; ${ }^{3}$ Department of Pathology, Third Medical Faculty, Charles University, Prague, Czech Republic
}

Received November 17, 2011; Accepted January 27, 2012

DOI: $10.3892 /$ ijo.2012.1410

\begin{abstract}
Granulocyte-macrophage colony stimulating factor (GM-CSF) is considered to be the most effective immunostimulating factor for the construction of gene-engineered anti-cancer vaccines. In some tumour cells, this type of genetic modification has resulted in the loss of the oncogenic potential. This was not the case with bcr-abl-transformed mouse 12B1 cells. A cell line, designated 12B1/GM-CSF/cl-5 producing more than $100 \mathrm{ng} / 10^{6}$ cells/24 h, displayed higher pathogenicity than the parental, non-transduced cells. Although the tumours induced by the parental 12B1 cells and 12B1/GM-CSF/ cl-5 cells appeared nearly at the same time and then grew at an approximately equal rate, the latter mice were in a much poorer clinical condition. In these animals the growth of the tumours was associated with gradually increasing blood levels of GM-CSF. In both groups of animals splenomegaly was observed; it was much more pronounced in the case of 12B1/ GM-CSF/cl-5-inoculated animals. While in the case of animals inoculated with the parental cells the splenomegaly was probably mainly due to infiltration with tumour cells, in the animals inoculated with the GM-CSF-secreting cells splenomegaly and derangement of parenchymal organs, such as lungs, liver and kidneys, were more complex, including congestion and infiltration with hemopoietic cells, predominantly immature cells of myeloid lineage. The most conspicuous of these changes was the hyperaemia of the lungs. No such alterations were seen in animals inoculated with the parental cells. On the other hand, the contents of $\mathrm{T}$ regulatory cells were comparable in both groups and they increased in parallel at the end of the observation period. When GM-CSF neutralizing antibody was administered to animals inoculated with the 12B1/GM-CSF/ cl-5 cells, the pathological changes observed within the organs
\end{abstract}

Correspondence to: Dr Vladimír Vonka, Department of Experimental Virology, Institute of Hematology and Blood Transfusion, U Nemocnice 1, Prague, Czech Republic

E-mail:vonka@uhkt.cz

Key words: bcr-abl, gene-modified cells, granulocyte-macrophage colony stimulating factor, organ damage were suppressed, this proving that the overproduced GM-CSF and not any other substance, played the key role in their induction.

\section{Introduction}

The granulocyte-macrophage colony stimulating factor (GM-CSF) is a cytokine with pleiotropic effects on the immune system, capable of eliciting well co-ordinated cellular and humoral immune responses. It plays roles both in the priming and effector phases of the immune response. Since the demonstration that among tumour cells genetically engineered to express different cytokines those secreting GM-CSF are the most immunogenic $(1,2)$, tumour cell vaccines secreting this cytokine have run into the focus of interest. During the latest decade, inactivated GM-CSF secreting cancer vaccines have been used in numerous preclinical and clinical studies (reviewed in refs. 2-5).

However, several reports have suggested that GM-CSF is a dual-role player: it may either augment the anti-cancer response or suppress it. This apparently depends on the magnitude of its production. Convincing evidence has been obtained that the levels of GM-CSF exceeding a certain upper limit recruit myeloid-derived suppressor cells (MDSC) that facilitate tumour progression by suppressing both innate and adaptive immune reactions (reviewed in refs. 6-8) and by displaying proangiogenic activity (9). Furthermore, GM-CSF induces the expression of milk fat globule EGF-8 (MFG-E8), which suppresses anti-tumour immune reactions through a variety of mechanisms including the expansion of $\mathrm{T}$ regulatory cells (Tregs) $(10,11)$, and which has recently also been shown to promote the oncogenic potential of at least some cancer cells by stimulating their proliferation (12). Also some other studies have demonstrated that high GM-CSF production may have harmful consequences. It has been reported that an overexpression of GM-CSF in the lungs of rats resulted in pulmonary fibrosis (13). Transgenic mice with constitutive overproduction of GM-CSF exhibited damage to several organs, including eye, skeletal muscles and lungs, in which alveolar proteinosis and accumulation of lymphocytes in the peribronchial area were observed (14). In a more recent study with transgenic animals, the GM-CSF gene equipped with the promoter and 
flanking sequences of the human CRP gene was used (15). Hepatosplenomegaly with increased extramedullar haematopoiesis and high numbers of activated monocytes in the blood of the animals was observed; however, no changes in the lungs or kidneys were reported. The subsequent administration of LPS resulted in an extreme increase of circulating GM-CSF levels and rapid death of the animals owing to an endotoxic shock. Similar was the outcome of an experiment in which animals were injected intravenously with a high dose of recombinant adenovirus encoding murine GM-CSF (16). Adenoviruses are known to infect hepatocytes after intravenous inoculation. The inoculation of the recombinant adenovirus resulted in a transitory appearance of GM-CSF blood levels, hepatosplenomegaly and a massive accumulation of mononuclear cells in the liver. The administration of LPS on day 7 was followed by severe liver damage characterized by massive haemorrhagic injury and extensive apoptosis of hepatocytes associated with a marked increase of alanine aminotransferase (ALT). The authors concluded that the augmented susceptibility to LPS was a consequence of the accumulation of mononuclear cells in the liver. All animals died within $24 \mathrm{~h}$ after its administration. Damage to other organs was not reported.

Recently, we reported on some properties of the mouse bcr-abl-transformed, GM-CSF-secreting 12B1 cells (17). These cells not only retained their oncogenic potential, but also were apparently more pathogenic than the parental cells. Autopsy revealed alterations of a variety of organs. In the present report we describe the pathological changes induced by the 12B1/GM-CSF-secreting cells in individual organs and their development.

\section{Materials and methods}

Cell lines and media. 12B1 is a murine leukaemia cell line derived by the transformation of Balb/c bone marrow cells with a retrovirus-derived vector carrying the human bcr-abl (b3a2) fusion gene; the 12B1 cells are of early B cell lineage (18). They were kindly provided to us by E. Katsanis (University of Arizona, Tuscon, AZ). In vitro and in vivo properties of the 12B1 cells were described in more detail elsewhere $(19,20)$. More recent data indicate that they differ from the other bcr-abltransformed mouse cell lines in a number of other properties (Krmenčíková et al, unpublished). They express CD19 but are considerably bigger than the mature B-lymphocytes and have large segmented nuclei. These properties were utilized for identification of the tumour cells in the splenocyte population (see below). When administered intravenously, they induce acute leukaemia-like disease, with $1 \mathrm{TID}_{50}$ corresponding to approximately $10^{2}$ cells. After subcutaneous inoculation, 12B1 cells induce solid tumours, with $1 \mathrm{TID}_{50}$ corresponding to approximately $10^{2.5}$ cells. Cells were cultivated in RPMI-1640 medium (Sigma-Aldrich Corp., St. Louis, MO) supplemented with $10 \%$ heat-inactivated FCS (PAA Laboratories, Linz, Austria), $4 \mathrm{mM}$ glutamine, $1 \mathrm{mM}$ sodium pyruvate, $50 \mu \mathrm{M}$ 2-mercaptoethanol, penicillin $(100 \mathrm{U} / \mathrm{ml})$ and streptomycin $(100 \mu \mathrm{g} / \mathrm{ml})$ at $37^{\circ} \mathrm{C}$ in $5 \% \mathrm{CO}_{2}$ atmosphere. 12B1/GM-CSF/ cl-5 cells producing about $110 \mathrm{ng}$ of GM-CSF $/ 10^{6}$ cells $/ 24 \mathrm{~h}$, were isolated after electroporation with a plasmid carrying the genes for mouse GM-CSF and blasticidine resistance, as described (17). They were grown in medium supplemented with blasticidine ( $25 \mu \mathrm{g} / \mathrm{ml})$ (InvivoGen, San Diego, CA). In all experiments the third passage derived from a large frozen stock was used. For animal experiments, the cells were washed three times with PBS and the appropriate counts of cells in $0.2 \mathrm{ml}$ of PBS were injected subcutaneously.

Animal experiments. Six to eight-weeks old female Balb/c mice were obtained from Charles Rivers, Germany. All experiments were carried out in accordance with the Guidelines for Animal Experimentation valid in the Czech Republic. The 12B1 and 12B1/GM-CSF/cl-5 cells were washed three times with PBS and, if not indicated otherwise, counts of $5 \times 10^{3}$ in $0.2 \mathrm{ml}$ volumes of PBS were injected subcutaneously. The mice were monitored for symptoms of the disease and were sacrificed humanely. Blood samples were collected for determining the GM-CSF levels in the sera. At autopsy, several organs were taken for histopathological and immunohistochemical investigation. Mice were followed for up to 18 days.

To neutralize GM-CSF in inoculated mice, a monoclonal anti-mouse GM-CSF antibody (MP122E9, $0.5 \mathrm{mg} / \mathrm{ml}, \mathrm{R} \& \mathrm{D}$ Systems, Minneapolis, MN, USA) was used. Mice inoculated subcutaneously with $5 \times 10^{3} 12 \mathrm{~B} 1 / \mathrm{GM}-\mathrm{CSF} / \mathrm{cl}-5$ cells were divided into two groups, each group containing 3 animals. One group was treated with anti-GM-CSF antibody, while the other one remained untreated. Each animal in the first group received $100 \mu \mathrm{g}$ of anti-GM-CSF antibody on day 0 (i.e. simultaneously with cell administration) and $200 \mu \mathrm{g}$ on day 5. A third group of animal received only the antibody and no cells. The antibody was administered intraperitoneally. This experiment was terminated on day 14

Histology. Organs for histopathological investigation were fixed in $10 \%$ buffered formalin (in PBS). Paraffin-embedded samples were sliced using a microtome (Leova), to 3 to $5 \mu \mathrm{m}$ thick slices. After paraffin wash out, they were stained with hematoxylin and eosin (H\&E) (Dako, Denmark). The preparations derived from the kidneys were also stained with trichrome (Mallory Trichrome kit, Bamed, Czech Republic).

Immunohistochemistry. Sections of deparaffinised samples were incubated with rabbit anti-human ABL1 polyclonal antibody (pTyr272) (LifeSpan, Biosciences, Spain) diluted (1:50) for $1 \mathrm{~h}$. This antibody reacts with human but not with murine abl protein. Samples were washed with PBS, incubated with histidine (EXBIO, Czech Republic) for $30 \mathrm{~min}$ and subsequently treated with the Universal Immuno-Enzyme Polymer reagent Histofine (Nichrei Biosciences, Tokyo, Japan) following the manufacturer's instructions. DAB+ substrate chromogen (Dako) was used for visualization. Finally, cells were stained with hematoxylin-eosin (Dako).

Generation of cell cultures from tumours and organs infiltrated by tumour cells. Tumours and selected organs were removed from the mice under sterile conditions. They were mechanically disrupted in small volumes of complete RPMI medium to produce cell suspensions, which were filtered through a cell strainer and centrifuged. The cell pellets were resuspended and cultivated in regular media. Third passage of cell cultures was split into media with and without blasticidine. Growth activity and cytokine production were monitored. 
Measurement of cytokine production in sera and cell cultures. Sera from the inoculated animals were stored at $-20^{\circ} \mathrm{C}$ until investigation. Counts of $1 \times 10^{6}$ cells from cell cultures were seeded in $3 \mathrm{ml}$ medium. After $24 \mathrm{~h}$ they were spun down. The supernates were stored at $20^{\circ} \mathrm{C}$ until investigation. The concentration of GM-CSF in sera and media was determined by ELISA kit (BD OptEIA ${ }^{\mathrm{TM}}$ Set Mouse GM-CSF, BD Biosciences, San Diego, CA) according to the manufacturer's instructions.

Flow cytometry. Single-cell suspensions of splenocytes were prepared by mechanical disruption in a small volume of RPMI and filtration through a cell strainer. Red blood cells were removed by 5 -min treatment with ACK lysing buffer $(0.15 \mathrm{M}$ $\mathrm{NH}_{4} \mathrm{Cl}, 10 \mathrm{mM} \mathrm{KHCO}, 0.1 \mathrm{mM}$ EDTA) at room temperature. The cells were spun down and washed with RPMI $+2 \%$ FCS. Then $1 \times 10^{6}$ cells were incubated with $0.125 \mu \mathrm{g}$ of rat anti-mouseCD16/32 (BD Pharmingen, San Diego, CA) to block the Fc receptor and washed with FACS buffer (PBS, 2\% FCS, 0.09\% sodium azid). For cell phenotype characterization, the following antibodies were used: FITC-Gr-1 (eBioscience, San Diego, CA), PE-CD11b (BD Pharmingen), FITC-CD4 (BD Pharmingen), PE-Cy5-CD25, PE-CD19 (US Biologicals, Swampscott, MA) and PE-FoxP3 (eBioscience). The cells were incubated with cell-surface reacting antibodies for $30 \mathrm{~min}$ on ice in the dark. Then the cells were washed, fixed and permeabilized with BD Cytofix/Cytoperm Fixation/Permeabilization kit (BD Biosciences) according to the manufacturer's instructions. For the determination of Treg cells, the permeabilized cells, already labelled with CD4 and CD25 antibodies, were incubated $30 \mathrm{~min}$ with the PE-FoxP3 antibody. Flow cytometry was performed using Beckman Coulter EPICS XL. For analysis of the results FlowJo 7.6 software was used.

\section{Results}

Pathological changes in mice inoculated with 12B1/GM-CSF/ cl-5 cells. Mice inoculated subcutaneously with the 12B1/ GM-CSF/cl-5 cells and the parental 12B1 cells were followed for the development of tumours and pathological changes in various organs. Two mice from the 12B1/GM-CSF/cl-5-inoculated group and two mice from the 12B1-inoculated group were sacrificed at 2-day and 4-day intervals, respectively. In general, starting with day 6 , mice which had received the 12B1/GM-CSF/ cl-5 cells were in much poorer state than the 12B1-inoculated mice. They had bristled hair and exhibited weight loss. In these animals, macroscopic pathological changes were apparent at autopsy already on day 8. i.e. 4 days prior to the appearance of tumours. Among these, splenomegaly (Fig. 1A-c) and extensive foci of congestion of lungs (Fig. 1B-c) dominated. Such changes in lungs were not seen in animals inoculated with the parental 12B1 cells (Fig. 1B-b).

Tumour formation. Tumours were palpable from day 10 to 12 after cell inoculation in both groups and tumour growth did not significantly differ between mice inoculated with the parental 12B1 and the 12B1/GM-CSF/cl-5 cells (Fig. 2). Histologically, tumours in both groups were characterized by poorly differentiated cells with frequent mitoses. At the end of the observation period, a difference was encountered. While tumours induced by the 12B1/GM-CSF/cl-5 cells remained vital, extensive foci

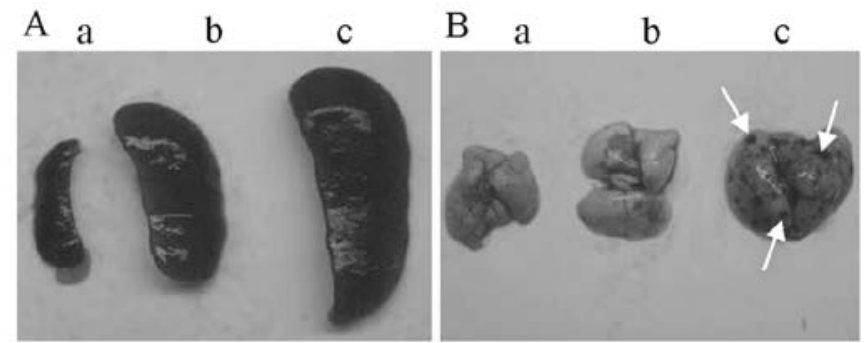

Figure 1. Spleens and lungs of mice inoculated with 12B1 and 12B1/GM-CSF/ cl-5 cells: differences in size and appearance. (A) Spleen (a) healthy mouse, (b) mouse inoculated with parental 12B1 cells, (c) mouse inoculated with 12B1/ GM-CSF/cl-5 cells. (B) Lung (a) healthy control mouse, (b) mouse inoculated with parental 12B1 cells, (c) mouse inoculated with 12B1/GM-CSF/cl-5 cells. Focuses of congestion are apparent (arrows). Day 18.

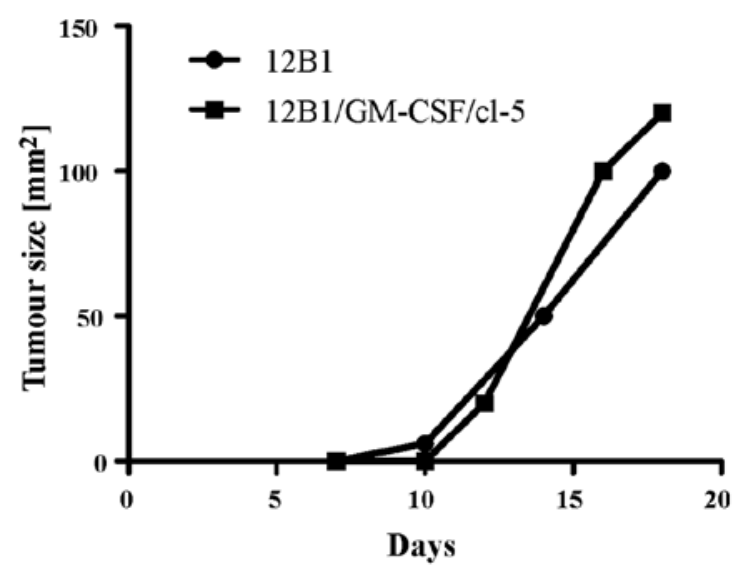

Figure 2. Tumour growth in mice inoculated with $12 \mathrm{~B} 1$ and 12B1/GM/cl-5 cells. Mice were inoculated subcutaneously with $5 \times 10^{3}$ cells. Tumour growth was monitored at two-day intervals. All animals eventually developed tumours.

of necrosis were seen in tumours induced by the parental cells (results not shown).

Serum levels of GM-CSF and its production by tumourderived cell cultures. The production of GM-CSF in sera of the inoculated animals was measured by ELISA. Throughout the observation period, GM-CSF was undetectable in sera of mice inoculated with parental 12B1 cells and in control healthy mice. In mice inoculated with $12 \mathrm{~B} 1 / \mathrm{GM}-\mathrm{CSF} / \mathrm{cl}-5$ cells, the level of GM-CSF in serum started to increase on day 8 and reached its peak, i.e. $280 \mathrm{ng} / \mathrm{ml}$, on day 16 (Fig. 3). Spleens and tumours of mice from this group of animals were taken on days 16 and 18 . Their portions were mechanically homogenized and the cells were cultivated in vitro in complete RPMI medium either with or without blasticidine. The cells grew very well in both media. After three passages, we collected the media and tested them in ELISA for the contents of GM-CSF. Cells derived from the spleens produced $83 \pm 12 \mathrm{ng} / 10^{6}$ cells $/ 24 \mathrm{~h}$ and cells derived from tumours produced $92 \pm 16 \mathrm{ng} / 10^{6}$ cells $/ 24 \mathrm{~h}$, indicating that the ability of the cells to secrete the cytokine remained essentially unchanged in the course of their growth in vivo.

Changes in spleens and composition of splenocyte populations. In both groups of inoculated animals marked splenomegaly was 


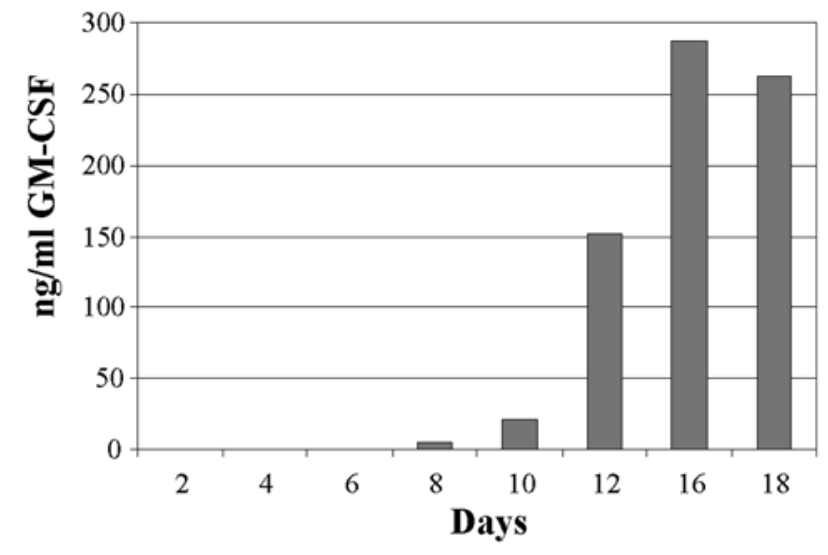

Figure 3. GM-CSF levels in sera of mice inoculated with 12B1/GM-CSF/cl-5 cells as determined by ELISA. Sera from two animals sacrificed on the days indicated were pooled. GM-CSF levels were undetectable in sera from healthy mice or mice inoculated with parental 12B1 cells.

observed from day 8 . The presence of GM-CSF in the blood of mice that had received the 12B1/GM-CSF/cl-5 cells correlated with their spleen enlargement. By the end of the observation period, the size of the spleens showed a nearly 20 -fold increase over the spleens of healthy animals and it also considerably surpassed the size of spleens of animals inoculated with the parental 12B1 cells. Spleens from the latter animals showed approximately only a 6 -fold increase over spleen size of healthy animals.

Results of the analysis of the splenocyte populations are shown in Figs. 4 and 5. As indicated in Fig. 4A and B in mice inoculated with GM-CSF producing cells there was a gradual increase of $\mathrm{CD}_{11 b^{+}}$cells. From day 6 , the population of $\mathrm{Grl}^{+} \mathrm{CD} 11 \mathrm{~b}^{+}$started to expand. Between day 2 and 18 their content increased from 2 to $21 \%$ and of $\mathrm{Grl}^{-} \mathrm{CD}^{-11 b^{+}}$cells from 2 to $34 \%$ of spleen cells. In the case of spleen cells from mice inoculated with parental 12B1, only a mild increase of cells with these markers was observed on day 16 and 18, respectively. By the end of the observation period the $\mathrm{Grl}^{+} \mathrm{CD} 11 \mathrm{~b}^{+}$ cells represented only $3.9 \%$ of spleen cells and the population

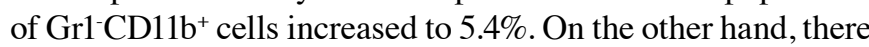
was little difference between the two groups of animals in the percentage of $\mathrm{CD} 4{ }^{+} \mathrm{CD} 25^{+} \mathrm{FoxP} 3^{+}$regulatory cells (Treg) within the $\mathrm{CD} 4^{+}$population. Until day 16 similarly low percentages of Tregs were detected in both groups and their relative count did not differ from Treg counts in healthy animals. Only on day 18 , i.e. at the end of the observation period, there was a marked but similar increase in Tregs in both parental 12B1-inoculated and 12B1/GM-CSF/cl-5-inoculated animals (Fig. 4C). To get basic information on the rate of infiltration of the spleens by the tumour cells, at the end of the observation period the splenocytes were also tested for their presence. The results are shown in Fig. 5. They suggest that the spleens of the two groups of animals were infiltrated with tumour cells to a different degree. On day 18 tumour cells represented $40 \%$ of the cells isolated from the spleens of mice inoculated with the parental cells but only $5 \%$ of cells isolated from the spleens of mice inoculated with 12B1/GM-CSF/cl-5 cells. To further confirm these data the spleens were investigated immunohistochemically making
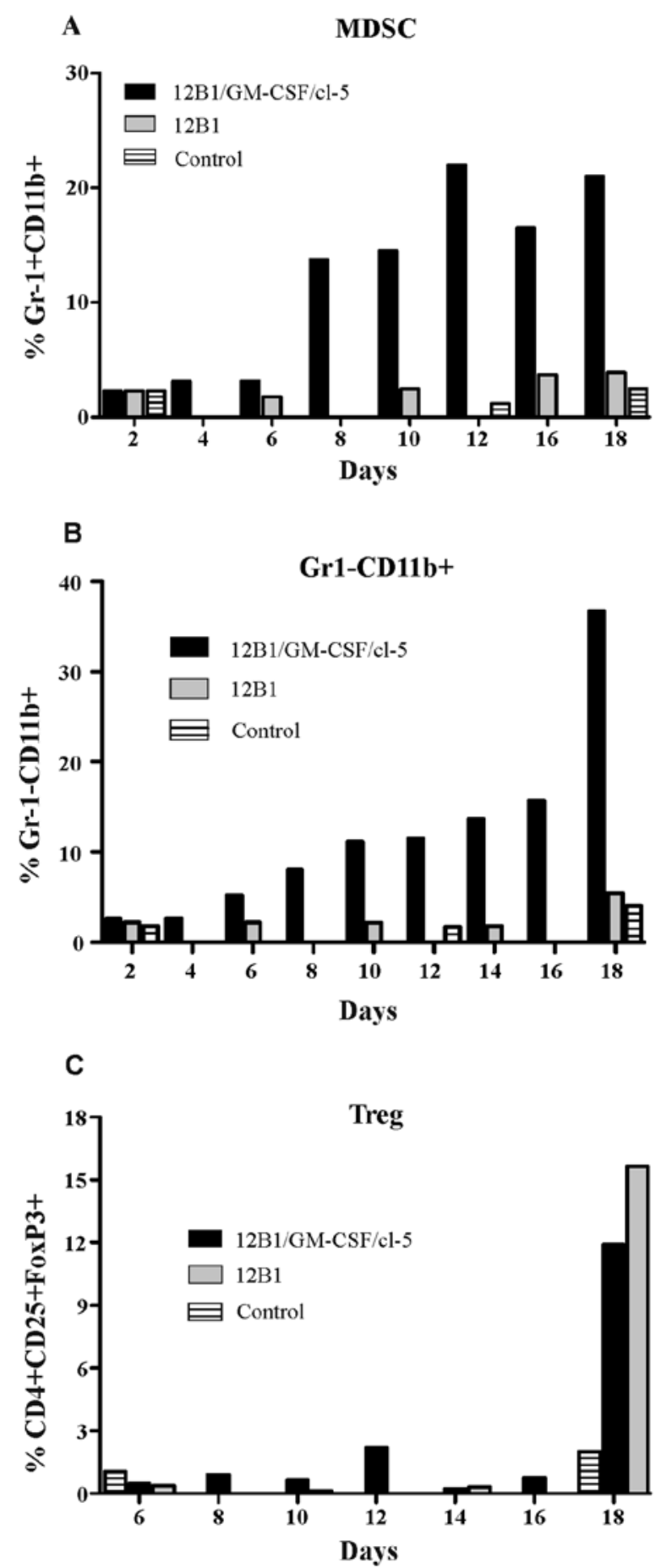

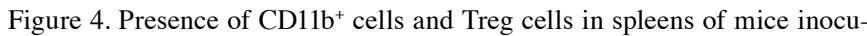
lated with $12 \mathrm{~B} 1$ and 12B1/GM-CSF/cl-5 cells as determined by FACS. (A) $\mathrm{Grl}^{+} \mathrm{CD} 11 \mathrm{~b}^{+}$cells, (B) $\mathrm{Grl}^{-} \mathrm{CD} 11 \mathrm{~b}^{+}$cells, (C) $\mathrm{CD} 4^{+} \mathrm{CD} 25^{+} \mathrm{FoxP}^{+}$cells within CD4 ${ }^{+}$Tcell subset. Day 18 .

use of an antibody reactive with human but not murine abl protein. The results are shown in Fig. 6. No reactive cells were detected in spleens of healthy animals. They were much more frequent in mice inoculated with parental cells than in those which received GM-CSF-producing cells.

Histological investigation of the spleens disclosed normal white and red pulp without marked congestion in animals 
A

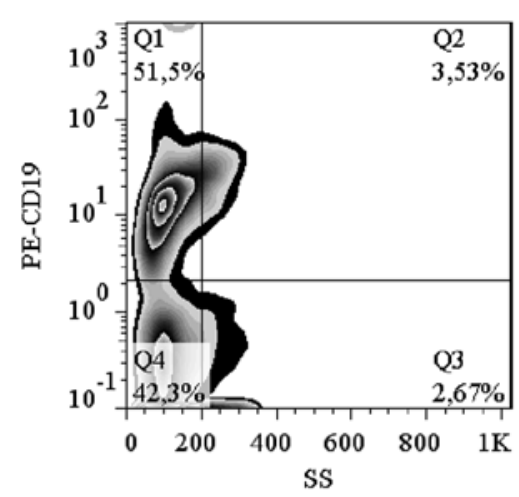

B

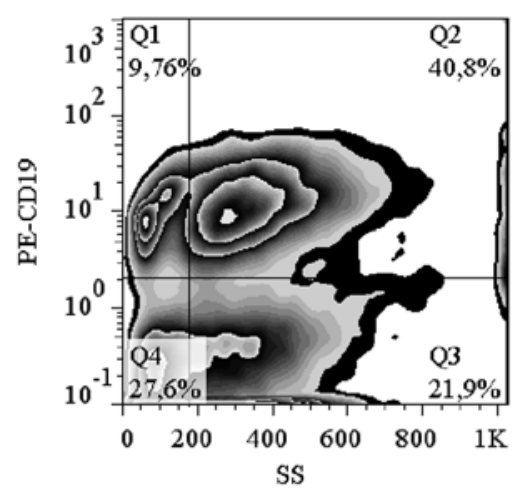

C 12B1/GM-CSF/cl-5

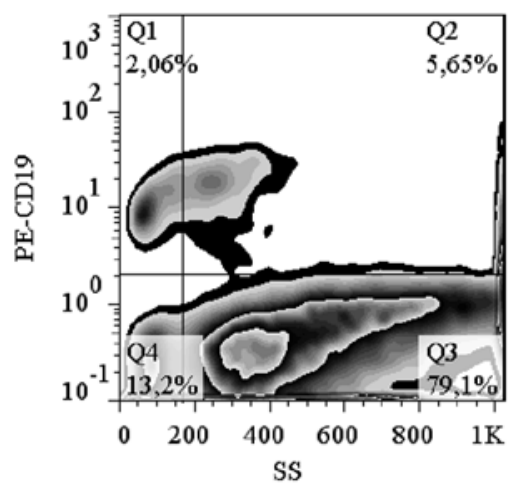

Figure 5. Presence of tumour cells in spleens from mice inoculated with 12B1 and 12B1/GM-CSF/cl-5 cells as determined by FACS at the end of the observation period. The results are presented as density plots. B-lymphocytes are gated in quadrant $1(\mathrm{Q} 1)$ and tumour cells in quadrant 2 (Q2) on the basis of side scatter and CD19 positivity. (A) Healthy control mouse, (B) mouse inoculated with parental 12B1 cells, (C) mouse inoculated with 12B1/GM-CSF/cl-5 cells. Day 18.

A

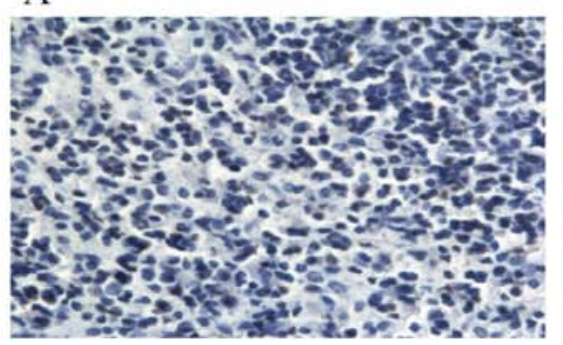

B

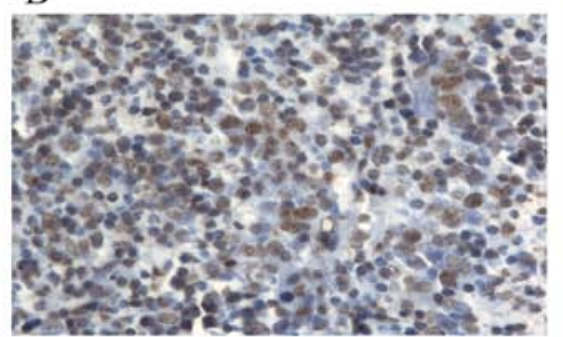

C

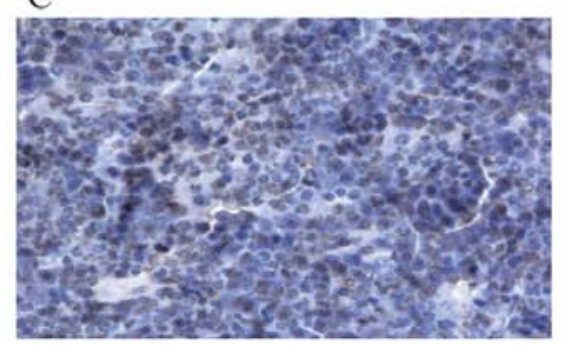

Figure 6. Immunohistochemical investigation of spleens of mice inoculated with 12B1 and 12B1/GM-CSF/cl-5 cells. For detection of tumour cells carrying human bcr-abl gene, rabbit antibody reactive with human but not with murine abl protein was employed. Histofine Universal Immuno-enzyme Polymer Reagent and DAB substrate chromogen were used for visualization. (A) Healthy control mouse, (B) mouse inoculated with 12B1 cells, (C) reduced number of positive tumour cells in mouse inoculated with 12B1/GM-CSF/cl-5 cells. Day 18.

inoculated with the parental 12B1 (Fig. 7A-a). On the contrary, the spleens of 12B1/GM-CSF/cl-5-inoculated animals exhibited marked changes in their structure. The red pulp was congested, expanded and largely replaced the white pulp, and extramedullary haematopoiesis became much more apparent. Multiple megakaryocytes were detected (Fig. 7A-b and c).

Changes in lungs. From day 10, marked alterations were observed in the lungs of mice inoculated with 12B1/GM-CSF/ cl-5 cells. As already mentioned, macroscopical inspection revealed focuses of congestion already at the autopsy. Histological examination of lungs of mice inoculated with the parental 12B1 cells did not reveal any structural changes of the lung parenchyma (Fig. 7B-a). On the other hand, the lungs in 12B1/GM-CSF/cl-5-inoculated mice displayed morphological alterations. Interalveolar septa were extended due to congestion and infiltration with immature hematopoietic cells was apparent. Focal collapse and pneumorrhagia were also present (Fig. 7B-b and c).

Changes in livers. In mice inoculated with 12B1/GM-CSF/cl-5 cells, hepatomegaly accompanied with irregular hemorrhagic foci and other changes was observed. On the other hand, the structure of liver in animals inoculated with the parental 12B1 cells remained unaffected (Fig. 7C-a). Fig. 7C-b and c shows the liver of an animal inoculated with 12B1/GM-CSF/cl-5 cells. In the latter, prominent congestion and focal haemorrhage were found. Apart from focal infiltrates of tumour cells, massive infiltration of liver parenchyma with hematopoietic cells resembling a myeloproliferative syndrome was also present. Reactive changes of hepatocytes manifested themselves by a variability of the size of the nuclei and binuclear hepatocytes were observed.

Changes in kidneys. Kidneys of animals inoculated with the parental cells were free of any marked pathological changes (Fig. 7D-a). At the autopsy, the first changes of kidneys in animals inoculated with the 12B1/GM-CSF/cl-5 cells were observed on day 12 . They were characterized by congestion and focal haemorrhages and progressed until the end of the observation period. Histological examination discovered congestion, higher cellularity of glomeruli and focal infiltrates with haematopoietic cells, predominantly of immature myeloid lineage (Fig. 7D-b). Proximal tubuli displayed massive deposition of hyaline droplets as revealed by trichrome staining (Fig. 7D-c).

Neutralization of GM-CSF by specific anti-GM-CSF antibody. To prove that the pathological changes had been induced by overexpressed GM-CSF, the GM-CSF-neutralization test was performed as described in the Materials and methods. Lungs 
12B1

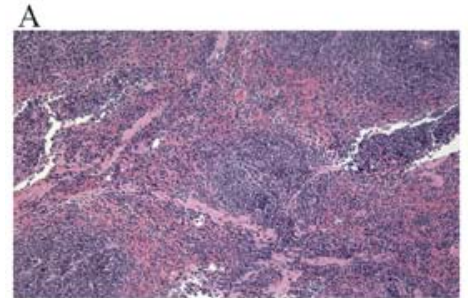

a

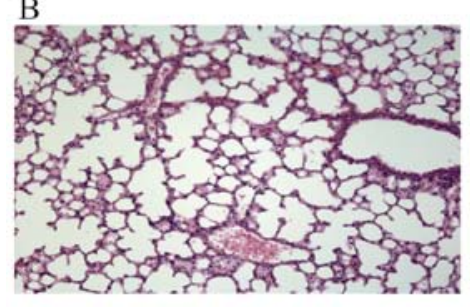

a

C

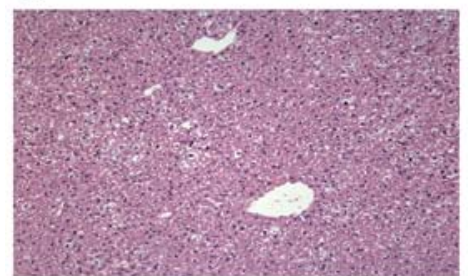

a

D

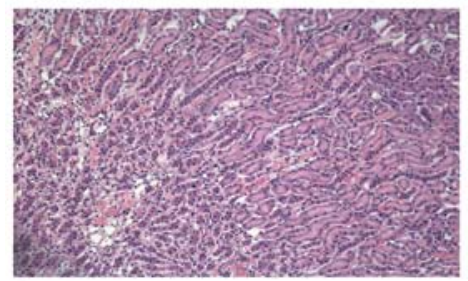

a
12B1/GM-CSF/cl-5

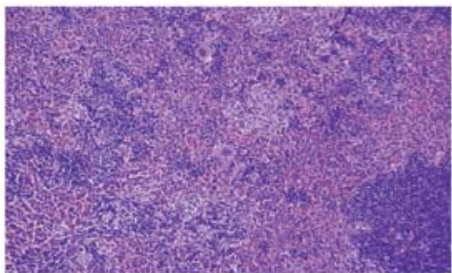

b

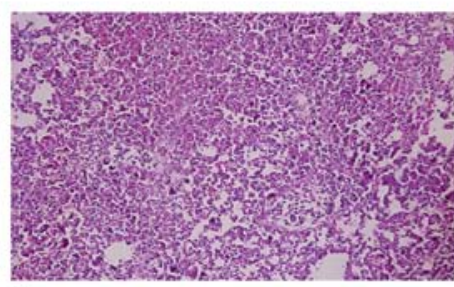

b

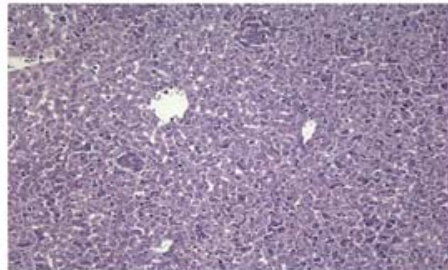

b

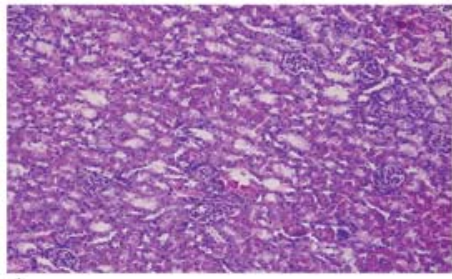

b

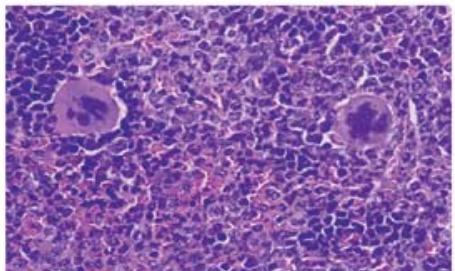

c
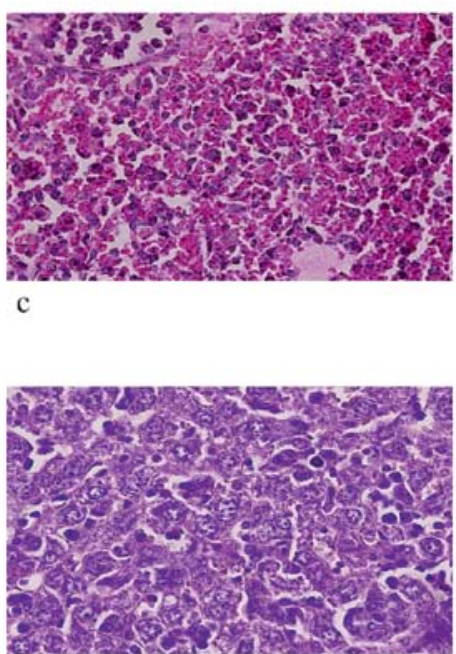

c

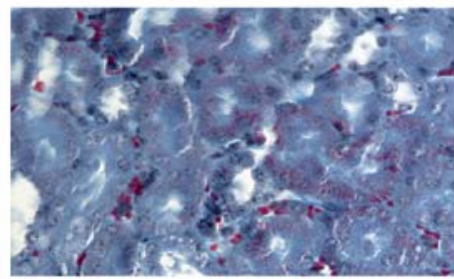

$\mathrm{c}$

Figure 7. Histological investigation of various organs. (A) (a) Spleen of a mouse inoculated with parental 12B1 cells (H\&E, 100x). (b) Spleen of a mouse inoculated with 12B1/GM-CSF/cl-5 cells (H\&E, 100x). Congestion, extensive foci of extramedullary hematopoiesis, reduction of white pulp. (c) Spleen of a mouse inoculated with 12B1/GM-CSF/cl-5 cells (H\&E, 400x). Detail of extramedullary hematopoiesis with megakaryocytes and immature myeloid cells. Day 16. (B) (a) Lung of a mouse inoculated with parental 12B1 cells (H\&E,100x). (b) Lung of a mouse inoculated with 12B1/GM-CSF/cl-5 cells (H\&E, 100x). Focal collapse of parenchyma with pneumorrhagia. (c) Lung of a mouse inoculated with 12B1/GM-CSF/cl-5 cells (H\&E, 400x). Immature myeloid cells within the lung parenchyma, focal pneumorrhagia. Day 16. (C) (a) Liver of a mouse inoculated with parental 12B1 cells (H\&E, 100x). (b) Liver of a mouse inoculated with 12B1/GM-CSF/cl-5 cells (H\&E, 100x). Small focal infiltrates of tumour cells and extramedullary hematopoiesis. (c) Liver of a mouse inoculated with 12B1/GM-CSF/cl-5 cells (H\&E, 400x). Extramedullary hematopoiesis within liver parenchyma, reactive changes of hepatocytes and immature myeloid cells in sinusoids. Day 16. (D) (a) Kidney of a mouse inoculated with parental 12B1 cells. (b) Kidney of a mouse inoculated with 12B1/GM-CSF/cl-5 cells (H\&E, 100x). Congestion, higher cellularity of glomeruli. (c) Kidney of a mouse inoculated with 12B1/GM-CSF/cl-5 cells (Mallory trichrome, 400x). Numerous hyaline droplets within epithelia of proximal tubules, focal interstitial infiltrates of immature myeloid cells. Day 12.

A

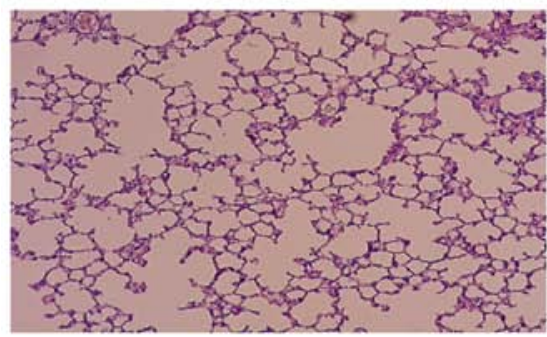

B

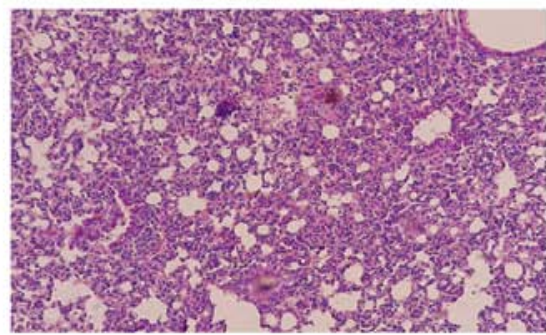

$\mathrm{C}$

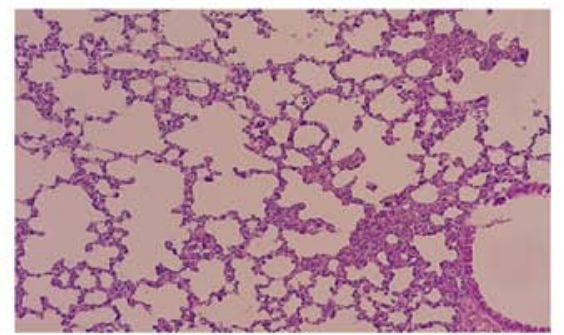

Figure 8. Lungs of mice inoculated with 12B1/GM-CSF/cl-5 cells: neutralization effect of antibody against mouse GM-CSF. (A) Lung of a mouse which did not receive the cells but was inoculated with GM-CSF-neutralizing antibody. (B) Lung of a mouse inoculated with 12B1/GM-CSF/cl-5 cells. (C) Lung of a mouse inoculated with 12B1/GM-CSF/cl-5 cells and treated with GM-CSF-neutralizing antibody. Day 16. 
of mice treated and non-treated with the GM-CSF antibody are shown in Fig. 8. Both of these animals possessed tumours of approximately the same size ( $8 \mathrm{~mm}$ in their longest diameter) at the time of autopsy. It is evident that the haemorrhagic changes characteristic for the 12B1/GM-CSF/cl-5-inoculated animals were essentially suppressed by the administration of the antibody. The extent of organ injury was also markedly reduced in spleens, livers and kidneys (data not shown). The findings were similar in all three animals which had been antibody-treated and in all three animals which had not been treated with the antibody. No pathological changes were detected in animals which had received only the antibody.

\section{Discussion}

We recently observed that, contrary to our expectation, the mouse bcr-abl-transformed 12B1 cells gene-engineered to secrete high amounts of GM-CSF did not lose their oncogenic potential, but in fact were more pathogenic than the parental cells (17). The pathogenicity of the GM-CSF-secreting cells was dependent on the amount of the cytokine produced, this suggesting that the cytokine itself might be responsible for the additional detrimental effects.

Although harmful effects of high doses of GM-CSF have repeatedly been reported, to the best of our knowledge no study describing them in various organs in some detail has been published until now. Since GM-CSF is the most frequently used cytokine for the preparation of vaccines based on inactivated gene-engineered tumour cells, we considered it useful to investigate its putative adverse effects more closely. For this purpose we employed the high producer cell clone designated 12B1/GM-CSF/cl-5 and compared its pathogenicity with the parental 12B1 cells. The ability of these two cell lines to induce subcutaneous tumours did not differ substantially, but there were marked differences in the clinical picture. However, other differences were also evident. The sera of animals inoculated with the GM-CSF-secreting cells, but not of those inoculated with the parental cells, contained detectable amounts of GM-CSF and its levels increased in parallel with the growth of the tumours and gradual spleen enlargement. The cell cultures derived from the tumours or organs infiltrated with tumour cells produced approximately the same amount of cytokine as did the cells which had been inoculated. Since this suggested that the production of the cytokine was unaltered during the growth of the cells in vivo, one has to assume that a tumour sized $1 \mathrm{~cm}^{3}$ produced up to $100 \mu \mathrm{g}$ of the cytokine per day. In animals inoculated with the gene-modified cells, but not in those inoculated with the parental cells, marked adverse effects on a variety of organs were detected. Possibly the most striking was the damage to lungs which even macroscopically exhibited strong congestion and focal bleeding. Histological investigation revealed complex changes characterized by congestion and thickening of interalveolar septa, focal collapse and pneumorrhagia. Thus, our findings differ from those reported by Metcalf et al (14). The difference may be associated with the lower levels of GM-CSF achieved in these authors' transgenic animals and also with the fact that macrophages and not rapidly replicating tumour cells were the major producer of the cytokine in their system. We also found prominent alterations of the spleens. Splenomegaly was detected in animals inoculated with both the parental and the GM-CSF-secreting cells; however, it was much more pronounced in the latter animals. In animals inoculated with the gene-modified but not in those inoculated with the parental cells, a gradual replacement of white pulp by the red pulp and a very extensive congestion associated with a substantially increased extramedullary haematopoiesis were seen. Originally, we had thought that the splenomegaly was due to the infiltration with tumour cells. However, this did not appear to be the case. As indicated by both FACS analysis and immunohistochemistry, tumour cells represented only a small fraction of splenocytes in animals inoculated with the GM-CSF secreting cells. The splenomegaly in animals inoculated with the parental cells was possibly largely due to the infiltration of this organ by tumour cells. An additional observation dealt with the different presence of immature myeloid cells among the splenocytes of the two groups of animals. It is known that GM-CSF can elicit a heterogeneous population of immature myeloid cells, designated MDSC, characterized by the $\mathrm{Grl}^{+} \mathrm{CD} 11 \mathrm{~b}^{+}$phenotype, the products of which impair immune responses. MDSC were much more frequent in animals inoculated with the GM-CSF-secreting cells which is in agreement with the earlier observations $(10,21)$. Also the $\mathrm{Grl}^{-} \mathrm{CD} 11 \mathrm{~b}^{+}$cells, representing a mixture of other immature myeloid cells were much more frequent in animals inoculated with the 12B1/GM-CSF/cl-5 cells than in animals inoculated with the parental cells. On the other hand, no marked difference was observed in the relative contents of Treg cells. This seems to be at variance with some earlier observations which have indicated that GM-CSF can support the expansion of Treg cells $(22,23)$, though our present observation may be obscured to a certain degree by the different sizes of the spleens. Other organs displaying pathological changes in the 12B1/GM-CSF/ cl-5-inoculated animals were the livers and the kidneys. In both of them congestion and focal haemorrhages were observed. Extramedullary haematopoiesis was detected in the liver. In the kidneys massive deposits of hyaline droplets within the proximal tubuli epithelia were seen. As far as we are aware, damage to kidneys in animals overexpressing GM-CSF has not previously been reported.

Thus, the direct adverse effect of the megadoses of GM-CSF, which were active in our experiments, apparently overshadowed the immunosuppressive activity of this cytokine, although one of the mechanisms responsible for the immunosuppression, i.e. the recruitment of MDSC, was also operative and most likely contributed to the pathological changes described. MDSC could also contribute to the effective vascularization of the tumours induced by the GM-CSF-secreting cells. At variance with the advanced tumours induced by the parental cells they were without necrotic foci.

Although the present results strongly suggested that overproduced GM-CSF was closely related to the pathological changes described in this report, there was a theoretical possibility that some other factor produced by the gene-engineered cells was involved. Therefore, we checked whether the development of the pathological changes could be suppressed by the administration of GM-CSF-neutralizing antibody to mice inoculated by the 12B1/GM-CSF/cl-5 cells. As the chief indicator of the antibody effect the extensive bleeding into lungs seen in these animals was chosen. The administration of the antibody nearly completely suppressed it and protective effects could also be 
seen in other organs monitored. This proved beyond reasonable doubt that GM-CSF was responsible for at least a great majority of the organ damage observed. Sublata causa, tollitur effectus.

Definitely, the present findings should not discourage those involved in the development of vaccines secreting GM-CSF, because the use of live vaccines for the immunotherapy of cancer patients is not under serious consideration. Still, the present data may invite some caution when planning the immunization schemes and when using recombinant GM-CSF along with other vaccines.

To summarize, in our experiments we observed substantial alterations of lungs, spleens, kidneys and livers caused by a high amount of GM-CSF secreted by the tumour cells. We demonstrated that the pathological changes observed were induced by this cytokine and were not due to some unintentional changes in the biology of the cells that might have been caused by their transduction or subsequent selection and cultivation.

\section{Acknowledgements}

We would like to thank Soňa Lísová for her help with histological analysis. This study was supported by the grant no. NS-10634-3/2009 the Internal Grant Agency of the Ministry of Health, Czech Republic and Research Project MŠMT MSM 0021620808 .

\section{References}

1. Dranoff G, Jaffee E, Lazenby A, et al: Vaccination with irradiated tumor cells engineered to secrete murine granulocyte-macrophage colony-stimulating factor stimulates potent, specific, and long-lasting anti-tumor immunity. Proc Natl Acad Sci USA 90: 3539-3543, 1993

2. Dranoff G: GM-CSF-based cancer vaccines. Immunol Rev 188: $147-154,2002$.

3. Eager R and Nemunaitis J: GM-CSF gene-transduced tumor vaccines. Mol Ther 12: 18-27, 2005.

4. Gupta R and Emens LA: GM-CSF-secreting vaccines for solid tumors: moving forward. Discov Med 10: 52-60, 2010.

5. Le DT, Pardoll DM and Jaffee EM: Cellular vaccine approaches. Cancer J 16: 304-310, 2010

6. Gabrilovich DI and Nagaraj S: Myeloid-derived suppressor cells as regulators of the immune system. Nat Rev Immunol 9: 162-174, 2009.

7. Youn JI and Gabrilovich DI: The biology of myeloid-derived suppressor cells: the blessing and the curse of morphological and functional heterogeneity. Eur J Immunol 40: 2969-2975, 2010.

8. Tadmor T, Attias D and Polliack A: Myeloid-derived suppressor cells-their role in haemato-oncological malignancies and other cancers and possible implications for therapy. $\mathrm{Br} \mathrm{J}$ Haematol 153 : 557-567, 2011.

9. Yang L, DeBusk LM, Fukuda K, et al: Expansion of myeloid immune suppressor $\mathrm{Gr}^{+} \mathrm{CD} 11 \mathrm{~b}^{+}$cells in tumor-bearing host directly promotes tumor angiogenesis. Cancer Cell 6: 409-421, 2004.
10. Serafini P, Carbley R, Noonan KA, Tan G, Bronte V and Borrello I: High-dose granulocyte-macrophage colony-stimulating factorproducing vaccines impair the immune response through the recruitment of myeloid suppressor cells. Cancer Res 64: 6337-6343, 2004.

11. Pan PY, Ma G, Weber KJ, et al: Immune stimulatory receptor $\mathrm{CD} 40$ is required for T-cell suppression and T regulatory cell activation mediated by myeloid-derived suppressor cells in cancer. Cancer Res 70: 99-108, 2010.

12. Carrascosa C, Obula RG, Missiaglia E, et al: MFG-E8/lactadherin regulates cyclins D1/D3 expression and enhances the tumorigenic potential of mammary epithelial cells. Oncogene: Aug 15, 2011 (Epub ahead of print). doi: 10.1038/onc.2011.356.

13. Xing Z, Tremblay GM, Sime PJ and Gauldie J: Overexpression of granulocyte-macrophage colony-stimulating factor induces pulmonary granulation tissue formation and fibrosis by induction of transforming growth factor-beta 1 and myofibroblast accumulation. Am J Pathol 150: 59-66, 1997.

14. Metcalf D, Mifsud S, Di RL, Robb L, Nicola NA and Alexander W: The biological consequences of excess GM-CSF levels in transgenic mice also lacking high-affinity receptors for GM-CSF Leukemia 12: 353-362, 1998.

15. Burke B, Pridmore A, Harraghy N, Collick A, Brown J and Mitchell T: Transgenic mice showing inflammation-inducible overexpression of granulocyte macrophage colony-stimulating factor. Clin Diagn Lab Immunol 11: 588-598, 2004.

16. Hirano K, Shimizu Y, Nakayama Y, Minemura M, Yasumura S and Sugiyama T: Overexpression of granulocyte-macrophage colony-stimulating factor in mouse liver enhances the susceptibility of lipopolysaccharide leading to massive apoptosis of hepatocytes. Liver Int 25: 1027-1035, 2005.

17. Petrackova M, Tachezy R and Vonka V: Properties of bcr-abltransformed mouse 12B1 cells secreting interleukin 2 (IL-2) and granulocyte-macrphage colony stimulating factor (GM-CSF): I. Derivation, genetic stability, oncogenicity and immunogenicity. Int J Oncol 40: 1668-1676, 2012.

18. McLaughlin J, Chianese E and Witte ON: In vitro transformation of immature hematopoietic cells by the P210 BCR/ABL oncogene product of the Philadelphia chromosome. Proc Natl Acad Sci USA 84: 6558-6562, 1987.

19. Sobotkova E, Ludvikova V, Petrackova M, et al: Characteristic of two mouse bcr-abl-transformed cell lines: I. General properties of the cells. Folia Biol (Praha) 51: 12-18, 2005.

20. Jelinek F, Sobotkova E and Vonka V: Characteristics of two mouse bcr-abl-transformed cell lines. II. Pathological lesions induced in mice. Folia Biol (Praha) 51: 93-102, 2005.

21. Finke J, Ko J, Rini B, Rayman P, Ireland J and Cohen P: MDSC as a mechanism of tumor escape from sunitinib mediated antiangiogenic therapy. Int Immunopharmacol 11: 856-861, 2011.

22. Vasu C, Dogan RN, Holterman MJ and Prabhakar BS: Selective induction of dendritic cells using granulocyte macrophage-colony stimulating factor, but not fms-like tyrosine kinase receptor 3-ligand, activates thyroglobulin-specific $\mathrm{CD}^{+} / \mathrm{CD} 25^{+} \mathrm{T}$ cells and suppresses experimental autoimmune thyroiditis. J Immunol 170: 5511-5522, 2003.

23. Bhattacharya P, Gopisetty A, Ganesh BB, Sheng JR and Prabhakar BS: GM-CSF-induced, bone-marrow-derived dendritic cells can expand natural Tregs and induce adaptive Tregs by different mechanisms. J Leukoc Biol 89: 235-249, 2011. 